\title{
ANTI-COMMUTING REAL HYPERSURFACES IN COMPLEX TWO-PLANE GRASSMANNIANS
}

\author{
IMSOON JEONG, HYUN JIN LEE and YOUNG JIN SUH ${ }^{\bowtie}$
}

(Received 17 October 2007)

\begin{abstract}
In this paper we give a nonexistence theorem for real hypersurfaces in complex two-plane Grassmannians $G_{2}\left(\mathbb{C}^{m+2}\right)$ with anti-commuting shape operator.

2000 Mathematics subject classification: primary 53C40; secondary 53C15.

Keywords and phrases: complex two-plane Grassmannians, hypersurfaces of type $B$, anti-commuting, contact hypersurface.
\end{abstract}

\section{Introduction}

In the geometry of real hypersurfaces in complex space forms $M_{m}(c)$ or in quaternionic space forms there have been many characterizations of model hypersurfaces of type $A_{1}, A_{2}, B, C, D$ and $E$ in complex projective space $P_{m}(\mathbb{C})$, of type $A_{0}, A_{1}, A_{2}$ and $B$ in complex hyperbolic space $H_{m}(\mathbb{C})$, or of type $A_{1}, A_{2}$ and $B$ in quaternionic projective space $\mathbb{H} P^{m}$, which are completely classified by Cecil and Ryan [5], Kimura [6], Berndt [1], and Martinez and Pérez [7], respectively. Among them there have been only a few characterizations of homogeneous hypersurfaces of type $B$ in complex projective space $P_{m}(\mathbb{C})$. For example, the condition that $A \phi+\phi A=k \phi$, for nonzero constant $k$, is a model characterization of this kind of type $B$, which is a tube over a real projective space $\mathbb{R} P^{n}$ in $P_{m}(\mathbb{C}), m=2 n$ (see Yano and Kon [9]).

Let $M$ be a $(4 m-1)$-dimensional Riemannian manifold with an almost contact structure $(\phi, \xi, \eta)$ and an associated Riemannian metric $g$. Write

$$
\omega(X, Y)=g(\phi X, Y)
$$

where $\omega$ defines a 2-form on $M$ and $\operatorname{rank} \omega=\operatorname{rank} \phi=4 m-2$.

The first and the third authors are supported by grant Project No. R17-2008-001-01001-0 from KOSEF and the second author by grant Project No. KRF-2007-355-C00004 from KRF.

(c) 2008 Australian Mathematical Society 0004-9727/08 \$A2.00+0.00 
If there is a nonzero-valued function $\rho$ such that

$$
\rho g(\phi X, Y)=\rho \omega(X, Y)=d \eta(X, Y)
$$

the rank of the matrix $(\omega)$ being $4 m-2$,

$$
\eta \wedge \overbrace{\omega \wedge \cdots \wedge \omega}^{2 m-1 \text { times }}=\eta \wedge \rho^{-(2 m-1)} \overbrace{d \eta \wedge \cdots \wedge d \eta}^{2 m-1 \text { times }} \neq 0 .
$$

Let us denote by $G_{2}\left(\mathbb{C}^{m+2}\right)$ the set of all complex two-dimensional linear subspaces of $\mathbb{C}^{m+2}$. We call such a set $G_{2}\left(\mathbb{C}^{m+2}\right)$ complex two-plane Grassmannians. This Riemannian symmetric space $G_{2}\left(\mathbb{C}^{m+2}\right)$ has a remarkable geometry that is equipped with both a Kähler structure $J$ and a quaternionic Kähler structure $\mathfrak{J}=$ $\operatorname{Span}\left\{J_{1}, J_{2}, J_{3}\right\}$ not containing $J$. In other words, $G_{2}\left(\mathbb{C}^{m+2}\right)$ is the unique compact, irreducible, Kähler, quaternionic Kähler manifold which is not a hyperkähler manifold (see Berndt and Suh [3, 4]).

Now we consider a $(4 m-1)$-dimensional real hypersurface $M$ in complex twoplane Grassmannians $G_{2}\left(\mathbb{C}^{m+2}\right)$. Then from the Kähler structure of $G_{2}\left(\mathbb{C}^{m+2}\right)$ there exists an almost contact structure $\phi$ on $M$. If the nonzero function $\rho$ satisfies (0.2), we call $M$ a contact hypersurface of the Kähler manifold. Moreover, it can easily be proved that a real hypersurface $M$ in $G_{2}\left(\mathbb{C}^{m+2}\right)$ is contact if and only if there exists a nonzero constant function $\rho$ defined on $M$ such that

$$
\phi A+A \phi=k \phi, \quad k=2 \rho .
$$

This means that

$$
g((\phi A+A \phi) X, Y)=2 d \eta(X, Y)
$$

where the exterior derivative $d \eta$ of the 1 -form $\eta$ is defined by

$$
d \eta(X, Y)=\left(\nabla_{X} \eta\right) Y-\left(\nabla_{Y} \eta\right) X
$$

for any vector fields $X, Y$ on $M$ in $G_{2}\left(\mathbb{C}^{m+2}\right)$.

On the other hand, in $G_{2}\left(\mathbb{C}^{m+2}\right)$ we are able to consider two kinds of natural geometric condition for real hypersurfaces $M$ that

$$
[\xi]=\operatorname{Span}\{\xi\} \quad \text { or } \quad \mathfrak{D}^{\perp}=\operatorname{Span}\left\{\xi_{1}, \xi_{2}, \xi_{3}\right\}, \quad \xi_{i}=-J_{i} N, \quad i=1,2,3
$$

where $N$ denotes a unit normal to $M$, is invariant under the shape operator $A$ of $M$ in $G_{2}\left(\mathbb{C}^{m+2}\right)$. The first result in this direction is the classification of real hypersurfaces in $G_{2}\left(\mathbb{C}^{m+2}\right)$ satisfying both conditions. Namely, Berndt and Suh [3] have proved the following. 
THEOREM A. Let $M$ be a connected real hypersurface in $G_{2}\left(\mathbb{C}^{m+2}\right), m \geq 3$. Then both $[\xi]$ and $\mathfrak{D}^{\perp}$ are invariant under the shape operator of $M$ if and only if:

(A) $\quad M$ is an open part of a tube around a totally geodesic $G_{2}\left(\mathbb{C}^{m+1}\right)$ in $G_{2}\left(\mathbb{C}^{m+2}\right)$; or

(B) $m$ is even, say $m=2 n$, and $M$ is an open part of a tube around a totally geodesic $\mathbb{H} P^{n}$ in $G_{2}\left(\mathbb{C}^{m+2}\right)$.

In Theorem A the vector $\xi$ contained in the one-dimensional distribution $[\xi]$ is said to be a Hopf vector when it becomes a principal vector for the shape operator $A$ of $M$ in $G_{2}\left(\mathbb{C}^{m+2}\right)$. Moreover, in such a situation $M$ is said to be a Hopf hypersurface. Besides this, a real hypersurface $M$ in $G_{2}\left(\mathbb{C}^{m+2}\right)$ also admits the three-dimensional distribution $\mathfrak{D}^{\perp}$, which is spanned by almost contact three-structure vector fields $\left\{\xi_{1}, \xi_{2}, \xi_{3}\right\}$, such that $T_{x} M=\mathfrak{D} \oplus \mathfrak{D}^{\perp}$. Also Berndt and Suh [4] have given a characterization of real hypersurfaces of type $A$ when the shape operator $A$ of $M$ in $G_{2}\left(\mathbb{C}^{m+2}\right)$ commutes with the structure tensor $\phi$, which is equivalent to the condition that the Reeb flow on $M$ is isometric, as follows.

THEOREM B. Let $M$ be a connected orientable real hypersurface in $G_{2}\left(\mathbb{C}^{m+2}\right)$, $m \geq 3$. Then the Reeb flow on $M$ is isometric if and only if $M$ is an open part of a tube around a totally geodesic $G_{2}\left(\mathbb{C}^{m+1}\right)$ in $G_{2}\left(\mathbb{C}^{m+2}\right)$.

On the other hand, as a characterization of real hypersurfaces of type $B$ in complex two-plane Grassmannians $G_{2}\left(\mathbb{C}^{m+2}\right)$ in Theorem A, Suh [8], asserted the following fact in terms of the contact hypersurface.

TheOREM C. Let $M$ be a real hypersurface in $G_{2}\left(\mathbb{C}^{m+2}\right)$ with constant mean curvature satisfying

$$
A \phi+\phi A=k \phi
$$

where the function $k$ is nonzero and constant. Then $M$ is congruent to an open part of a tube around a totally geodesic $\mathbb{H} P^{n}$ in $G_{2}\left(\mathbb{C}^{m+2}\right)$, where $m=2 n$.

Now in this paper let us consider a real hypersurface $M$ in the complex two-plane Grassmannian $G_{2}\left(\mathbb{C}^{m+2}\right)$ satisfying $A \phi+\phi A=0$. When the function $k$ mentioned in Theorem $\mathrm{C}$ identically vanishes, the shape operator is said to be anti-commuting, that is, the shape operator $A$ of $M$ in $G_{2}\left(\mathbb{C}^{m+2}\right)$ satisfies

$$
A \phi+\phi A=0
$$

In such a case we call a real hypersurface $M$ in $G_{2}\left(\mathbb{C}^{m+2}\right)$ satisfying (*) an anticommuting hypersurface. We give a nonexistence property of hypersurfaces in $G_{2}\left(\mathbb{C}^{m+2}\right)$ with anti-commuting shape operator as follows.

THEOREM. There exist no anti-commuting real hypersurfaces in $G_{2}\left(\mathbb{C}^{m+2}\right)$ with constant mean curvature. 


\section{Riemannian geometry of $G_{2}\left(\mathbb{C}^{m+2}\right)$}

In this section we summarize basic material about $G_{2}\left(\mathbb{C}^{m+2}\right)$; for details we refer to [2-4]. By $G_{2}\left(\mathbb{C}^{m+2}\right)$ we denote the set of all complex two-dimensional linear subspaces in $\mathbb{C}^{m+2}$. The special unitary group $G=S U(m+2)$ acts transitively on $G_{2}\left(\mathbb{C}^{m+2}\right)$ with stabilizer isomorphic to $K=S(U(2) \times U(m)) \subset G$. Then $G_{2}\left(\mathbb{C}^{m+2}\right)$ can be identified with the homogeneous space $G / K$, which we equip with the unique analytic structure for which the natural action of $G$ on $G_{2}\left(\mathbb{C}^{m+2}\right)$ becomes analytic. Denote by $\mathfrak{g}$ and $\mathfrak{k}$ the Lie algebra of $G$ and $K$, respectively, and by $\mathfrak{m}$ the orthogonal complement of $\mathfrak{k}$ in $\mathfrak{g}$ with respect to the Cartan-Killing form $B$ of $\mathfrak{g}$. Then $\mathfrak{g}=\mathfrak{k} \oplus \mathfrak{m}$ is an $A d(K)$-invariant reductive decomposition of $\mathfrak{g}$. We put $o=e K$ and identify $T_{o} G_{2}\left(\mathbb{C}^{m+2}\right)$ with $\mathfrak{m}$ in the usual manner. Since $B$ is negative definite on $\mathfrak{g}$, its negative restricted to $\mathfrak{m} \times \mathfrak{m}$ yields a positive definite inner product on $\mathfrak{m}$. By $\operatorname{Ad}(K)$ invariance of $B$ this inner product can be extended to a $G$-invariant Riemannian metric $g$ on $G_{2}\left(\mathbb{C}^{m+2}\right)$. In this way $G_{2}\left(\mathbb{C}^{m+2}\right)$ becomes a Riemannian homogeneous space, even a Riemannian symmetric space. For computational reasons we normalize $g$ such that the maximal sectional curvature of $\left(G_{2}\left(\mathbb{C}^{m+2}\right), g\right)$ is 8 . Since $G_{2}\left(\mathbb{C}^{3}\right)$ is isometric to the three-dimensional complex projective space $\mathbb{C} P^{3}$ with constant holomorphic sectional curvature 8 , we shall assume that $m \geq 2$ from now on. Note that the isomorphism $\operatorname{Spin}(6) \simeq S U(4)$ yields an isometry between $G_{2}\left(\mathbb{C}^{4}\right)$ and the real Grassmann manifold $G_{2}^{+}\left(\mathbb{R}^{6}\right)$ of oriented two-dimensional linear subspaces of $\mathbb{R}^{6}$.

The Lie algebra $\mathfrak{k}$ has the direct sum decomposition $\mathfrak{k}=\mathfrak{s} u(m) \oplus \mathfrak{s} u(2) \oplus \Re$, where $\mathfrak{R}$ is the center of $\mathfrak{k}$. Viewing $\mathfrak{k}$ as the holonomy algebra of $G_{2}\left(\mathbb{C}^{m+2}\right)$, the center $\mathfrak{R}$ induces a Kähler structure $J$ and the $\mathfrak{s} u(2)$-part a quaternionic Kähler structure $\mathfrak{J}$ on $G_{2}\left(\mathbb{C}^{m+2}\right)$. If $J_{1}$ is any almost Hermitian structure in $\mathfrak{J}$, then $J J_{1}=J_{1} J$, and $J J_{1}$ is a symmetric endomorphism with $\left(J J_{1}\right)^{2}=I$ and $\operatorname{tr}\left(J J_{1}\right)=0$. This fact will be used frequently throughout this paper.

A canonical local basis $J_{1}, J_{2}, J_{3}$ of $\mathfrak{J}$ consists of three local almost Hermitian structures $J_{v}$ in $\mathfrak{J}$ such that $J_{v} J_{v+1}=J_{v+2}=-J_{v+1} J_{v}$, where the index is taken modulo 3. Since $\mathfrak{J}$ is parallel with respect to the Riemannian connection $\bar{\nabla}$ of $\left(G_{2}\left(\mathbb{C}^{m+2}\right), g\right)$, there exist for any canonical local basis $J_{1}, J_{2}, J_{3}$ of $\mathfrak{J}$ three local 1 -forms $q_{1}, q_{2}, q_{3}$ such that

$$
\bar{\nabla}_{X} J_{v}=q_{v+2}(X) J_{v+1}-q_{v+1}(X) J_{v+2}
$$

for all vector fields $X$ on $G_{2}\left(\mathbb{C}^{m+2}\right)$.

Let $p \in G_{2}\left(\mathbb{C}^{m+2}\right)$ and $W$ be a subspace of $T_{p} G_{2}\left(\mathbb{C}^{m+2}\right)$. We say that $W$ is a quaternionic subspace of $T_{p} G_{2}\left(\mathbb{C}^{m+2}\right)$ if $J W \subset W$ for all $J \in \mathfrak{J}_{p}$. And we say that $W$ is a totally complex subspace of $T_{p} G_{2}\left(\mathbb{C}^{m+2}\right)$ if there exists a one-dimensional subspace $\mathfrak{V}$ of $\mathfrak{J}_{p}$ such that $J W \subset W$ for all $J \in \mathfrak{V}$ and $J W \perp W$ for all $J \in \mathfrak{V}^{\perp} \subset$ $\mathfrak{J}_{p}$. Here, the orthogonal complement of $\mathfrak{V}$ in $\mathfrak{J}_{p}$ is taken with respect to the bundle metric and orientation on $\mathfrak{J}$ for which any local oriented orthonormal frame field of $\mathfrak{J}$ is a canonical local basis of $\mathfrak{J}$. A quaternionic (or totally complex) submanifold of 
$G_{2}\left(\mathbb{C}^{m+2}\right)$ is a submanifold all of whose tangent spaces are quaternionic (or totally complex) subspaces of the corresponding tangent spaces of $G_{2}\left(\mathbb{C}^{m+2}\right)$.

The Riemannian curvature tensor $\bar{R}$ of $G_{2}\left(\mathbb{C}^{m+2}\right)$ is locally given by

$$
\begin{aligned}
\bar{R}(X, Y) Z= & g(Y, Z) X-g(X, Z) Y+g(J Y, Z) J X \\
& -g(J X, Z) J Y-2 g(J X, Y) J Z \\
& +\sum_{\nu=1}^{3}\left\{g\left(J_{v} Y, Z\right) J_{v} X\right. \\
& \left.-g\left(J_{v} X, Z\right) J_{v} Y-2 g\left(J_{v} X, Y\right) J_{v} Z\right\} \\
& +\sum_{\nu=1}^{3}\left\{g\left(J_{v} J Y, Z\right) J_{v} J X-g\left(J_{v} J X, Z\right) J_{v} J Y\right\},
\end{aligned}
$$

where $J_{1}, J_{2}, J_{3}$ is any canonical local basis of $\mathfrak{J}$.

\section{Some fundamental formulas}

In this section let us give some basic formulas for real hypersurfaces in $G_{2}\left(\mathbb{C}^{m+2}\right)$ which will be used later.

The Kähler structure $J$ of $G_{2}\left(\mathbb{C}^{m+2}\right)$ induces on $M$ an almost contact metric structure $(\phi, \xi, \eta, g)$. Furthemore, let $J_{1}, J_{2}, J_{3}$ be a canonical local basis of $\mathfrak{J}$. Then expression (1.2) for the curvature tensor $\bar{R}$, the Gauss and the Codazzi equations are respectively given by

$$
\begin{aligned}
R(X, Y) Z= & g(Y, Z) X-g(X, Z) Y \\
& +g(\phi Y, Z) \phi X-g(\phi X, Z) \phi Y-2 g(\phi X, Y) \phi Z \\
& +\sum_{\nu=1}^{3}\left\{g\left(\phi_{\nu} Y, Z\right) \phi_{\nu} X-g\left(\phi_{\nu} X, Z\right) \phi_{\nu} Y-2 g\left(\phi_{\nu} X, Y\right) \phi_{\nu} Z\right\} \\
& +\sum_{\nu=1}^{3}\left\{g\left(\phi_{\nu} \phi Y, Z\right) \phi_{\nu} \phi X-g\left(\phi_{\nu} \phi X, Z\right) \phi_{\nu} \phi Y\right\} \\
& -\sum_{\nu=1}^{3}\left\{\eta(Y) \eta_{\nu}(Z) \phi_{\nu} \phi X-\eta(X) \eta_{\nu}(Z) \phi_{\nu} \phi Y\right\} \\
& -\sum_{\nu=1}^{3}\left\{\eta(X) g\left(\phi_{\nu} \phi Y, Z\right)-\eta(Y) g\left(\phi_{\nu} \phi X, Z\right)\right\} \xi_{\nu} \\
& +g(A Y, Z) A X-g(A X, Z) A Y
\end{aligned}
$$

and

$$
\begin{aligned}
\left(\nabla_{X} A\right) Y-\left(\nabla_{Y} A\right) X= & \eta(X) \phi Y-\eta(Y) \phi X-2 g(\phi X, Y) \xi \\
& +\sum_{\nu=1}^{3}\left\{\eta_{\nu}(X) \phi_{\nu} Y-\eta_{\nu}(Y) \phi_{\nu} X-2 g\left(\phi_{\nu} X, Y\right) \xi_{\nu}\right\}
\end{aligned}
$$




$$
\begin{aligned}
& +\sum_{\nu=1}^{3}\left\{\eta_{\nu}(\phi X) \phi_{\nu} \phi Y-\eta_{\nu}(\phi Y) \phi_{\nu} \phi X\right\} \\
& +\sum_{\nu=1}^{3}\left\{\eta(X) \eta_{\nu}(\phi Y)-\eta(Y) \eta_{\nu}(\phi X)\right\} \xi_{\nu},
\end{aligned}
$$

where $R$ denotes the curvature tensor of a real hypersurface $M$ in $G_{2}\left(\mathbb{C}^{m+2}\right)$.

The following identities can be proved straightforwardly and will be used frequently in subsequent calculations:

$$
\begin{gathered}
\phi_{v+1} \xi_{v}=-\xi_{v+2}, \quad \phi_{v} \xi_{v+1}=\xi_{v+2} \\
\phi \xi_{v}=\phi_{v} \xi, \quad \eta_{v}(\phi X)=\eta\left(\phi_{v} X\right), \\
\phi_{v} \phi_{v+1} X=\phi_{v+2} X+\eta_{v+1}(X) \xi_{v} \\
\phi_{v+1} \phi_{v} X=-\phi_{v+2} X+\eta_{v}(X) \xi_{v+1}
\end{gathered}
$$

Now let us put

$$
J X=\phi X+\eta(X) N, \quad J_{v} X=\phi_{\nu} X+\eta_{v}(X) N
$$

for any tangent vector $X$ of a real hypersurface $M$ in $G_{2}\left(\mathbb{C}^{m+2}\right)$, where $N$ denotes a unit normal vector of $M$ in $G_{2}\left(\mathbb{C}^{m+2}\right)$. Then from this and formulas (1.1) and (2.1),

$$
\begin{gathered}
\left(\nabla_{X} \phi\right) Y=\eta(Y) A X-g(A X, Y) \xi, \quad \nabla_{X} \xi=\phi A X \\
\nabla_{X} \xi_{v}=q_{v+2}(X) \xi_{v+1}-q_{v+1}(X) \xi_{v+2}+\phi_{v} A X \\
\left(\nabla_{X} \phi_{v}\right) Y=-q_{v+1}(X) \phi_{v+2} Y+q_{v+2}(X) \phi_{v+1} Y+\eta_{v}(Y) A X-g(A X, Y) \xi_{v} .
\end{gathered}
$$

Summing up these formulas, we obtain

$$
\begin{aligned}
\nabla_{X}\left(\phi_{\nu} \xi\right)= & \nabla_{X}\left(\phi \xi_{v}\right) \\
= & \left(\nabla_{X} \phi\right) \xi_{v}+\phi\left(\nabla_{X} \xi_{v}\right) \\
= & q_{v+2}(X) \phi_{v+1} \xi-q_{v+1}(X) \phi_{v+2} \xi+\phi_{\nu} \phi A X \\
& -g(A X, \xi) \xi_{v}+\eta\left(\xi_{v}\right) A X .
\end{aligned}
$$

Moreover, from $J J_{v}=J_{v} J, v=1,2,3$, it follows that

$$
\phi \phi_{\nu} X=\phi_{\nu} \phi X+\eta_{\nu}(X) \xi-\eta(X) \xi_{v}
$$

\section{Some key propositions}

Now let us take an inner product to Codazzi's equation with $\xi$ and use (2.1) and (2.2). Then

$$
\begin{aligned}
& g\left(\left(\nabla_{X} A\right) Y, \xi\right)-g\left(\left(\nabla_{Y} A\right) X, \xi\right)=-2 g(\phi X, Y) \\
& \quad+2 \sum_{\nu=1}^{3}\left\{\eta_{\nu}(X) \eta_{\nu}(\phi Y)-\eta_{\nu}(Y) \eta_{\nu}(\phi X)-g\left(\phi_{\nu} X, Y\right) \eta_{\nu}(\xi)\right\}
\end{aligned}
$$


On the other hand, from formula (*) in the introduction, $A \xi=\alpha \xi$ where $\alpha=\eta(A \xi)$. From this, by taking the covariant derivative and using (2.2),

$$
\left(\nabla_{X} A\right) \xi=(X \alpha) \xi+\alpha \phi A X-A \phi A X .
$$

It follows that

$$
g\left(\left(\nabla_{X} A\right) \xi, Y\right)-g\left(\left(\nabla_{Y} A\right) \xi, X\right)=(X \alpha) \eta(Y)-(Y \alpha) \eta(X)-2 g(A \phi A X, Y) .
$$

Combining the above two equations,

$$
\begin{aligned}
& -2 g(\phi X, Y)+2 \sum_{\nu=1}^{3}\left\{\eta_{\nu}(X) \eta_{\nu}(\phi Y)-\eta_{v}(Y) \eta_{v}(\phi X)-g\left(\phi_{v} X, Y\right) \eta_{v}(\xi)\right\} \\
& =(X \alpha) \eta(Y)-(Y \alpha) \eta(X)-2 g(A \phi A X, Y)
\end{aligned}
$$

Putting $X=\xi$ in (3.1),

$$
\begin{gathered}
Y \alpha=(\xi \alpha) \eta(Y)-4 \sum_{\nu=1}^{3} \eta_{\nu}(\xi) \eta_{\nu}(\phi Y), \\
\operatorname{grad} \alpha=(\xi \alpha) \xi+4 \sum_{\nu=1}^{3} \eta_{\nu}(\xi) \phi \xi_{v} .
\end{gathered}
$$

Now substituting (3.2) into (3.1) gives

$$
\begin{gathered}
g(A \phi A X, Y)-g(\phi X, Y)=2 \sum_{\nu=1}^{3}\left\{\eta(X) \eta_{\nu}(\phi Y)-\eta(Y) \eta_{\nu}(\phi X)\right\} \eta_{\nu}(\xi) \\
-\sum_{\nu=1}^{3}\left\{\eta_{\nu}(X) \eta_{\nu}(\phi Y)-\eta_{\nu}(Y) \eta_{\nu}(\phi X)-g\left(\phi_{\nu} X, Y\right) \eta_{\nu}(\xi)\right\}
\end{gathered}
$$

for any tangent vector fields $X$ and $Y$ on $M$.

LEMMA 3.1. Let $M$ be a real hypersurface in $G_{2}\left(\mathbb{C}^{m+2}\right)$ with anti-commuting shape operator. Then $\operatorname{Tr} A=\alpha$.

PROOF. From (*) and (2.2) it follows that

$$
A X-\phi A \phi X-\alpha \eta(X) \xi=0,
$$

where we have put $\alpha=\eta(A \xi)$. If we take an orthonormal basis for $M$ in such a way that

$$
\left\{e_{i} \mid i=1,2, \ldots, 4 m-1\right\}
$$

then

$$
\sum_{i=1}^{4 m-1}\left\{g\left(A e_{i}, e_{i}\right)-g\left(\phi A \phi e_{i}, e_{i}\right)-\alpha \eta\left(e_{i}\right) g\left(\xi, e_{i}\right)\right\}=0,
$$

that is, $\operatorname{Tr} A-\operatorname{Tr} \phi A \phi-\alpha=0$. 
On the other hand, we see that $\operatorname{Tr} \phi A \phi=\operatorname{Tr} A \phi^{2}=-\operatorname{Tr} A+\alpha$. Therefore, $\operatorname{Tr} A=\alpha$.

LEMMA 3.2. Let $M$ be an anti-commuting real hypersurface in $G_{2}\left(\mathbb{C}^{m+2}\right)$ with constant mean curvature. Then $\xi$ belongs to either the distribution $\mathfrak{D}$ or the distribution $\mathfrak{D}^{\perp}$.

Proof. By Lemma 3.1 and the assumption we know that $\alpha$ is constant. And from (3.2) we get

$$
\sum_{\nu=1}^{3} \eta_{\nu}(\xi) \eta_{\nu}(\phi Y)=0
$$

Now let us put $\xi=\eta\left(X_{0}\right) X_{0}+\eta\left(\xi_{1}\right) \xi_{1}$ for some unit $X_{0} \in \mathfrak{D}$ and $\xi_{1} \in \mathfrak{D}^{\perp}$. Then

$$
\eta_{1}(\xi) \eta_{1}(\phi Y)=0 \text {. }
$$

First, if $\eta_{1}(\xi)=0$, then obviously $\xi \in \mathfrak{D}$.

Next let us consider the case where $\eta_{1}(\phi Y)=0$. By putting $\phi_{1} \xi$ in $Y$ we know $\eta\left(X_{0}\right)=0$, which gives $\xi \in \mathfrak{D}^{\perp}$. This proves our assertion.

Now let us denote by $\mathfrak{h}$ the orthogonal complement of the Reeb vector field $\xi$ in the tangent space of $M$ in $G_{2}\left(\mathbb{C}^{m+2}\right)$.

Lemma 3.3. If $A \phi+\phi A=0, X \in \mathfrak{h}$ with $A X=\lambda X$, then

$$
\lambda A \phi X-\phi X+\sum_{\nu=1}^{3}\left\{2 \eta_{\nu}(\xi) \eta_{\nu}(\phi X) \xi-\eta_{\nu}(X) \phi_{\nu} \xi-\eta_{\nu}(\phi X) \xi_{\nu}-\eta_{\nu}(\xi) \phi_{\nu} X\right\}=0
$$

PROOF. From (3.4) it follows that

$$
\begin{aligned}
A \phi A X & -\phi X+2 \sum_{\nu=1}^{3}\left\{\eta(X) \phi \xi_{v}+\eta_{v}(\phi X) \xi\right\} \eta_{v}(\xi) \\
& -\sum_{\nu=1}^{3}\left\{\eta_{v}(X) \phi \xi_{v}+\eta_{v}(\phi X) \xi_{v}+\eta_{v}(\xi) \phi_{v} X\right\}=0 .
\end{aligned}
$$

And using the assumption, for $X \in \mathfrak{h}$ such that $A X=\lambda X$, leads to the above formula.

PROPOSITION 3.4. There exist no anti-commuting real hypersurfaces in $G_{2}\left(\mathbb{C}^{m+2}\right)$ with constant mean curvature for $\xi \in \mathfrak{D}^{\perp}$.

Proof. By (3.5) and (*), for any $X \in \mathfrak{h}$,

$$
\left(\lambda^{2}+1\right) X-\sum_{\nu=1}^{3}\left\{\eta_{\nu}(X) \phi \phi_{\nu} \xi+\eta_{v}(\phi X) \phi \xi_{v}+\eta_{\nu}(\xi) \phi \phi_{\nu} X\right\}=0 .
$$


Since $\xi \in \mathfrak{D}^{\perp}$, we can put $\xi=\xi_{1}$. Then

$$
\left(\lambda^{2}+1\right) X+2 \eta_{2}(X) \xi_{2}+2 \eta_{3}(X) \xi_{3}-\phi \phi_{1} X=0 .
$$

Since $X \in \mathfrak{h}$, we suppose that $X=\mathfrak{D} X+\eta_{2}(X) \xi_{2}+\eta_{3}(X) \xi_{3}$. This implies that

$$
\left(\lambda^{2}+1\right) \mathfrak{D} X+\left(\lambda^{2}+2\right) \eta_{2}(X) \xi_{2}+\left(\lambda^{2}+2\right) \eta_{3}(X) \xi_{3}-\phi \phi_{1} \mathfrak{D} X=0 .
$$

Putting $X=\xi_{2}$ and $X=\xi_{3}$ in (3.6), we obtain $\left(\lambda^{2}+2\right) \xi_{2}=0$ and $\left(\lambda^{2}+2\right) \xi_{3}=$ 0 , respectively. From these facts, we see that $\lambda^{2}+2=0$. Therefore we get a contradiction, which gives the proof of our proposition.

\section{Anti-commuting hypersurfaces in $G_{2}\left(\mathbb{C}^{m+2}\right)$ for $\xi \in \mathfrak{D}^{\perp}$}

In this section we wish to show that there exist no hypersurfaces $M$ in $G_{2}\left(\mathbb{C}^{m+2}\right)$ with anti-commuting shape operator for $\xi \in \mathfrak{D}$. In order to do this we assert the following result.

LEMMA 4.1. Let $M$ be an anti-commuting real hypersurface in $G_{2}\left(\mathbb{C}^{m+2}\right)$ with constant mean curvature for $\xi \in \mathfrak{D}$. Then $g\left(A \mathfrak{D}, \mathfrak{D}^{\perp}\right)=0$.

PROOF. From the assumption we know that the function $\alpha$ is constant. Then for $\xi \in \mathfrak{D}$ and from (3.1), for any tangent vector field $X$ on $M$,

$$
\phi X-A \phi A X+\sum_{\nu=1}^{3}\left\{\eta_{\nu}(X) \phi_{\nu} \xi+\eta_{\nu}(\phi X) \xi_{\nu}\right\}=0 .
$$

To prove this lemma it suffices to show that $g\left(A \mathfrak{D}, \xi_{v}\right)=0, v=1,2,3$. In order to do this, we put

$$
\mathfrak{D}=[\xi] \oplus\left[\phi_{1} \xi, \phi_{2} \xi, \phi_{3} \xi\right] \oplus \mathfrak{D}_{0},
$$

where the distribution $\mathfrak{D}_{0}$ is an orthogonal complement of $[\xi] \oplus\left[\phi_{1} \xi, \phi_{2} \xi, \phi_{3} \xi\right]$ in the distribution $\mathfrak{D}$.

First, from the assumption $\xi \in \mathfrak{D}$ we know that $g\left(A \xi, \xi_{v}\right)=0, v=1,2,3$, because $A \xi=\alpha \xi$.

Next, we also get the conclusion $g\left(A \phi_{i} \xi, \xi_{v}\right)=0$, for $i, v=1,2,3$. In fact, using (2.3) and $\xi \in \mathfrak{D}$,

$$
\begin{aligned}
g\left(A \phi_{i} \xi, \xi_{v}\right) & =g\left(A \xi_{v}, \phi_{i} \xi\right) \\
& =g\left(A \xi_{v}, \phi \xi_{i}\right) \\
& =-g\left(\phi A \xi_{v}, \xi_{i}\right) \\
& =-g\left(\nabla_{\xi_{v}} \xi, \xi_{i}\right) \\
& =g\left(\xi, \nabla_{\xi_{v}} \xi_{i}\right) \\
& =g\left(\xi, q_{i+2}\left(\xi_{v}\right) \xi_{i+1}-q_{i+1}\left(\xi_{v}\right) \xi_{i+2}+\phi_{i} A \xi_{v}\right) \\
& =g\left(\xi, \phi_{i} A \xi_{v}\right) \\
& =-g\left(A \phi_{i} \xi, \xi_{v}\right)
\end{aligned}
$$

that is, $g\left(A \phi_{i} \xi, \xi_{v}\right)=0, v=1,2,3$. 
Finally, we consider the case $X \in \mathfrak{D}_{0}$, where the distribution $\mathfrak{D}_{0}$ is denoted by

$$
\mathfrak{D}_{0}=\left\{X \in \mathfrak{D} \mid X \perp \xi \text { and } \phi_{i} \xi, i=1,2,3\right\} .
$$

In order to show this, let us replace $X$ by $\xi_{\mu}$ in (4.1). Then it follows that

$$
2 \phi \xi_{\mu}=A \phi A \xi_{\mu}
$$

From this, together with the assumption (*),

$$
A^{2} \phi \xi_{\mu}=-2 \phi \xi_{\mu}
$$

Then multiplying both sides by $\phi$ and also using the formula $A \phi+\phi A=0$,

$$
A^{2}\left(-\xi_{\mu}+\eta\left(\xi_{\mu}\right) \xi\right)=-2\left(-\xi_{\mu}+\eta\left(\xi_{\mu}\right) \xi\right)
$$

This implies that

$$
A^{2} \xi_{\mu}=-2 \xi_{\mu}, \quad \mu=1,2,3
$$

On the other hand, if we consider the case where $X \in \mathfrak{D}_{0}$ in (3.4), then

$$
\phi X=A \phi A X
$$

From $A \phi+\phi A=0$, this becomes $-A^{2} \phi X=\phi X$. Then from this, replacing $X$ by $\phi X$ leads, for any $X \in \mathfrak{D}_{0}$, to

$$
A^{2} X=-X
$$

Using (4.2) and (4.3),

$$
\begin{aligned}
g\left(A X, \xi_{\mu}\right) & =g\left(A\left(-A^{2} X\right), \xi_{\mu}\right) \\
& =-g\left(A^{3} X, \xi_{\mu}\right)=-g\left(A X, A^{2} \xi_{\mu}\right) \\
& =-g\left(A X,-2 \xi_{\mu}\right)=2 g\left(A X, \xi_{\mu}\right),
\end{aligned}
$$

for any vector fields $X$ in $\mathfrak{D}_{0}$. Then for any $X \in \mathfrak{D}_{0}, g\left(A X, \xi_{\mu}\right)=0, \mu=1,2,3$. This completes the proof.

For a tube of type B in Theorem A let us recall a proposition given in Berndt and Suh [3] as follows.

Proposition A. Let $M$ be a connected real hypersurface of $G_{2}\left(\mathbb{C}^{m+2}\right)$. Suppose that $A \mathfrak{D} \subset \mathfrak{D}, A \xi=\alpha \xi$, and $\xi$ is tangent to $\mathfrak{D}$. Then the quaternionic dimension $m$ of $G_{2}\left(\mathbb{C}^{m+2}\right)$ is even, say $m=2 n$, and $M$ has five distinct constant principal curvatures

$$
\alpha=-2 \tan (2 r), \quad \beta=2 \cot (2 r), \quad \gamma=0, \quad \lambda=\cot (r), \quad \mu=-\tan (r),
$$


with some $r \in(0, \pi / 4)$. The corresponding multiplicities are

$$
m(\alpha)=1, \quad m(\beta)=3=m(\gamma), \quad m(\lambda)=4 n-4=m(\mu),
$$

and the corresponding eigenspaces are

$$
T_{\alpha}=\mathbb{R} \xi, \quad T_{\beta}=\mathfrak{J} J \xi, \quad T_{\gamma}=\mathfrak{J} \xi, \quad T_{\lambda}, \quad T_{\mu},
$$

where

$$
T_{\lambda} \oplus T_{\mu}=(\mathbb{H C} \xi)^{\perp}, \quad \mathfrak{J} T_{\lambda}=T_{\lambda}, \quad \mathfrak{J} T_{\mu}=T_{\mu}, \quad J T_{\lambda}=T_{\mu}
$$

Now by using Proposition A let us check whether a tube of type B in Theorem A, that is, a tube over a totally geodesic $\mathbb{H} P^{n}$ in $G_{2}\left(\mathbb{C}^{m+2}\right), m=2 n$ cannot satisfy the formula $(*)$.

In fact, for any $\xi_{v} \in T_{\beta}, \beta=2 \cot 2 r$, the eigenspace $T_{\gamma}=\mathfrak{J} \xi$ gives $\phi \xi_{v} \in T_{\gamma}$. This implies that $A \phi \xi_{v}=0$ for any $v=1,2,3$. From this,

$$
A \phi \xi_{v}+\phi A \xi_{v}=2 \cot 2 r \phi \xi_{v}=0
$$

For any $X \in T_{\lambda}, \lambda=\cot r$, we know that $J T_{\lambda}=T_{\mu}$ gives

$$
A \phi X+\phi A X=-\tan r \phi X+\cot r \phi X=2 \cot 2 r \phi X=0 .
$$

From this, we get $\cot 2 r=0$, giving a contradiction. So real hypersurfaces of type $\mathrm{B}$ cannot satisfy formula $(*)$.

PROPOSITION 4.2. There exist no anti-commuting real hypersurfaces in $G_{2}\left(\mathbb{C}^{m+2}\right)$ with constant mean curvature for $\xi \in \mathfrak{D}$.

Taking this Proposition 4.2 together with Proposition 3.4 gives a complete proof of our main theorem in the introduction.

\section{References}

[1] J. Berndt, 'Real hypersurfaces in quaternionic space forms', J. Reine Angew. Math. 419 (1991), 9-26.

[2] - 'Riemannian geometry of complex two-plane Grassmannians', Rend. Sem. Mat. Univ. Politec. Torino 55 (1997), 19-83.

[3] J. Berndt and Y. J. Suh, 'Real hypersurfaces in complex two-plane Grassmannians', Monatsh. Math. 127 (1999), 1-14.

[4] - 'Isometric flows on real hypersurfaces in complex two-plane Grassmannians', Monatsh. Math. 137 (2002), 87-98.

[5] T. E. Cecil and P. J. Ryan, 'Focal sets and real hypersurfaces in complex projective space', Trans. Amer. Math. Soc. 269 (1982), 481-499.

[6] M. Kimura, 'Real hypersurfaces and complex submanifolds in complex projective space', Trans. Amer. Math. Soc. 296 (1986), 137-149.

[7] A. Martinez and J. D. Pérez, 'Real hypersurfaces in quaternionic projective space', Ann. Mat. Pura Appl. 145 (1986), 355-384. 
[8] Y. J. Suh, 'Real hypersurfaces of type $B$ in complex two-plane Grassmannians', Monatsh. Math. 147 (2006), 337-355.

[9] K. Yano and M. Kon, CR-submanifolds of Kaehlerian and Sasakian Manifolds (Birkhäuser, Boston, Basel, Strutgart, 1983).

IMSOON JEONG, Department of Mathematics, Chungnam National University, Daejeon 305-764, Korea

e-mail: ijeong@nims.re.kr

HYUN JIN LEE, Department of Mathematics, Kyungpook National University, Taegu 702-701, Korea

e-mail: 1hjibis@yahoo.com

YOUNG JIN SUH, Department of Mathematics, Kyungpook National University, Taegu 702-701, Korea

e-mail: yjsuh@mail.knu.ac.kr 